Cinémas

Revue d'études cinématographiques

Journal of Film Studies

\title{
Présentation
}

\section{Édouard Arnoldy}

Volume 14, numéro 2-3, printemps 2004

Histoires croisées des images. Objets et méthodes

URI : https://id.erudit.org/iderudit/026002ar

DOI : https://doi.org/10.7202/026002ar

Aller au sommaire du numéro

Éditeur(s)

Cinémas

ISSN

1181-6945 (imprimé)

1705-6500 (numérique)

Découvrir la revue

Citer ce document

Arnoldy, É. (2004). Présentation. Cinémas, 14(2-3), 7-18.

https://doi.org/10.7202/026002ar d'utilisation que vous pouvez consulter en ligne.

https://apropos.erudit.org/fr/usagers/politique-dutilisation/ 


\section{Présentation}

\section{Édouard Arnoldy}

\section{L'histoire du cinéma se cherche}

Depuis les années 1980, au cours desquelles les pionniers de l'histoire du cinéma ont dû subir la fronde d'une jeune garde de "nouveaux historiens", un long chemin a été parcouru. Dès les faits d'armes de Brighton en 1978, les études cinématographiques menées aux États-Unis, au Québec et en Europe ont ébranlé le socle d'une discipline qui, forte de figures tutélaires, ne se posait jusqu'alors guère de questions sur elle-même, ses méthodes, ses objets. À tout le moins, peut-on considérer qu'il y a eu à ce moment un vacillement au sein d'un champ de recherche aux contours pourtant encore mal définis. Les conséquences de ce "tournant des années 1980 ", multiples et certes difficiles à évaluer, ne se résument pas aujourd'hui au partage d'un héritage ou d'un butin de guerre. Un constat, cependant, peut être dressé: celui de l'ouverture d'un champ d'études qui n'avait pas vraiment d'identité propre et qui donnait pourtant des signes avant-coureurs de repli sur luimême. Longtemps isolé de disciplines parfois proches, "le cinéma» est désormais un «objet d'étude» bien en place au sein d'institutions universitaires et de recherche, à mi-chemin entre l'histoire de l'art et les sciences de la communication. Surtout, de nouvelles exigences méthodologiques se sont ainsi affirmées. Ce qui s'est imposé, c'est la nécessité absolue de rompre avec la chronique cinématographique, plus ou moins soutenue, plus ou moins savante, pour faire place à une histoire du cinéma frappée du sceau de la rigueur scientifique et, parfois, doublée d'une réflexion théorique.

Aujourd'hui, le cinéma s'accorde au pluriel. C'est en effet devenu une évidence : le cinéma est, depuis ses lointaines 
origines, pris dans un réseau de pratiques les plus diverses, entre arts et médias, entre spectacle et beaux-arts, entre cultures noble et populaire. Enfin, il paraît maintenant y avoir unanimité quant à l'ambition d'aller au-delà des limites d'un champ (le cinéma) et de ses objets (les films). Du moins, les études cinématographiques cherchent-elles une posture pour mieux approcher le carrefour des séries culturelles et médiatiques où se situe assurément "le» cinéma. Insensiblement, des historiens et des théoriciens venus d'horizons divers se sont montrés de plus en plus intéressés par cet objet, étrange et singulier par la seule multiplicité de ses facettes - à la fois un divertissement et un art, une formidable machine à produire des capitaux et des idées. La discipline privilégie désormais les études transversales et sonde la mise en réseaux des multiples faisceaux qui font le cinéma. Autrement dit, il s'agit aujourd'hui de penser ensemble les dimensions multiples du cinéma et de son histoire. À partir de l'approche d'un fait culturel, un glissement s'est opéré dans le sens d'une histoire du cinéma de plus large portée, une histoire croisée des images.

L'histoire du cinéma est maintenant sur des rails qui la conduisent à s'interroger sur elle-même, à s'inquiéter de ses objets et de ses méthodes. Si des histoires clandestines et magistrales du cinéma coexistent désormais, si une histoire hétérogène, plurielle met aujourd'hui en doute une hypothétique autonomie du cinéma et d'aléatoires "spécificités cinématographiques", c'est bien parce que l'histoire du cinéma est en passe "d'excéder le cadre épistémologique de la discipline ${ }^{1}$ ". À l'avenir, les études cinématographiques paraissent devoir prioritairement s'intéresser aux possibles du cinéma. C'est exactement dans la foulée de ce mouvement que souhaite s'inscrire ce numéro de CiNéMAS. Les possibles du cinéma constituent précisément le centre de gravité de l'article de Maria Tortajada qui ouvre ce recueil. On a là, d'entrée, une ouverture exemplaire à la réflexion sur les histoires croisées des images. Ce texte dense et d'une rigueur indéfectible effectue l'un des trajets que ce numéro de CiNéMAS rêvait secrètement de voir s'accomplir. Si Tortajada utilise la voie d'une lecture critique de travaux empreints de l'archéologie foucaldienne, son programme constitue une 
réflexion théorique décisive qui n’a pas fini, osons le pari, de surprendre par sa fécondité et son amplitude ${ }^{2}$. Par la voie d'analyses textuelles particulièrement pointues, l'ambition affichée par Tortajada est bien d'aller au-delà du cinéma et du discours qu'il produit, pour mieux l'interroger, le comprendre, l'appréhender dans son extrême complexité. L'auteure revendique et explique ce choix:

En plongeant dans des recherches en rapport avec l'étude des médias (Kittler), de la littérature (Milner) ou avec une histoire de la vision et de la perception (Crary), approches qui d'ailleurs ne s'enferment pas strictement dans ces champs, cet article fait appel à des propositions permettant de décentrer le discours sur le cinéma: à des modèles, à des interrogations qu'il est possible de partager, des méthodes qu'il convient de s'approprier ou de transformer, etc. (p. 21)

Histoire, archéologie, épistémologie: ce parcours tout en mouvements, suis-je tenté d'écrire, permet de bien mesurer l'étendue des recherches autour de l'épistémè $e^{3}$ L'issue de l'entreprise de Tortajada est une percée débouchant sur un terrain largement en friche. Et sa visée est d'une envergure certaine: édifier les fondations de ce que l'auteure désigne par l'"épistémè cinéma autour de 1900 ", qui n’est plus tout à fait "l'histoire du cinéma» mais bien, écritelle, "un schème de relations signifiantes dans le contexte de l'époque et capable de modéliser une certaine approche du savoir et de l'expérience au-delà même des dispositifs de vision, mais précisément à partir d'eux".

À l'évidence, l'article de Tortajada devait ouvrir ce numéro, dans la mesure au moins où l'épistémologie traverse le numéro de part en part, parfois incidemment (Carl Havelange, Dick Tomasovic, Valérie Pozner), transversalement (Sylvie Lindeperg) ou de façon plus explicite (Mireille Berton, Thomas Elsaesser). Dans la foulée du texte de Tortajada, l'article de Mireille Berton constitue certainement une magnifique occasion de mesurer la portée et l'envergure de l'épistémè cinéma. Du moins cet article ne manque-t-il pas de voir autrement le cinéma - et, dans ce cas précis, ses liens à un puissant mode de pensée contemporain du septième art, la psychanalyse. Cet essai, dont on attend avec 
impatience les déploiements futurs, se caractérise par une hypothèse forte qui ose prendre le contre-pied de très nombreuses études proposant une lecture du cinéma à travers une grille psychanalytique. Berton ne souhaite pas la polémique et l'évite avec retenue. Pourtant, à la lecture de son article, on sonde peut-être mieux dans quelle mesure certaines analyses ou propositions théoriques — sans doute aveuglées par "la" psychanalyse (en parler au singulier est déjà réducteur) — n'ont finalement qu'une vision brouillée du cinéma et des films (voire de la psychanalyse elle-même). D'une relation "à sens unique" où la psychanalyse permet d'aider à la compréhension du cinéma, on passe à un rapport plus équitable, allant dans deux sens, du cinéma vers la psychanalyse et de la psychanalyse vers le cinéma ${ }^{4}$. Le postulat posé ici est précisément de prendre le recul nécessaire face à deux disciplines parfois confondues par trop de correspondances, pour mieux interroger le noud d'un réseau de relations où cinéma et psychanalyse se croisent vers 1900. La proposition est osée, et pas seulement parce qu'elle sème le trouble dans une relation presque gémellaire, trop lisse en apparence, défaite de toute aspérité. Car ce n'est pas cet aspect de l'article qui mérite d'être mis en exergue en ces lignes mais plutôt, comme pour Tortajada, une incessante circulation, ici entre psychanalyse, cinéma et épistémologie. "Au lieu d'entreprendre une lecture linéaire et "mécanique" de ces liens", Berton suggère "de les "désemboîter" afin de les ressaisir de manière plus articulée en croisant, au moyen d'une étude d'ordre épistémologique, deux histoires dans leurs fondements scientifiques et culturels». Encore une fois, il s'agit bien d'insister sur une rupture en train de s'opérer là, dans l'idée même que l'on peut se faire du cinéma et de son histoire. Ou : observer une discipline d'un autre œil - plus exactement selon des angles multiples —, rien de moins. Parvenant à gommer les ressemblances entre ces deux machines à penser, exactement contemporaines, toutes deux capables de rendre compte de l'imagination et du rêve avec une même puissance, l'étude entreprend avec audace de se glisser entre les mailles des liens tissés entre psychanalyse et cinéma dès l'aube du XX siècle. Et l'article a pour point de mire rien d'autre qu' « une histoire du cinéma 
soucieuse d'épistémologie» - soit une relecture en profondeur de «l'histoire du cinéma».

De son côté, Thomas Elsaesser propose de revenir sur les mutations considérables de l'histoire du cinéma et de son écriture, frappée sans cesse de plein fouet par quelques-uns des modes de pensée les plus féconds du XX siècle - dont l'archéologie de Foucault. Le débat ici établi entre Elsaesser et plusieurs auteurs de ce numéro de CiNéMAS permet de mieux jauger la prudence avec laquelle il s'agit de confronter l'archéologie et l'histoire du cinéma. Chacun souhaiterait sans doute interroger l'autre sur telle ou telle conséquence de la mutation épistémologique et des formations discursives mises en séries par Foucault. Pourtant, les différents articles s'accordent certainement pour dire que la discontinuité a autorisé l'histoire du cinéma à "penser la différence, [à] décrire des écarts et des dispersions, [à] dissocier la forme rassurante de l'identique" (Foucault). Elsaesser n'est pas loin de convenir de ce qui s'impose désormais: si l'histoire du cinéma est abordée dans son extrême complexité et si l'histoire du cinéma ose aujourd'hui revendiquer sa dispersion, c'est, me semble-t-il, parce que Foucault avait ouvert la voie — à propos d'objets tout autres. Dissertant sur cette dispersion salutaire, Elsaesser convie son lecteur à parcourir transversalement l'histoire du cinéma et à interroger l'intermédialité constitutive d'une pratique centenaire, "depuis toujours» à la croisée des médias. Et, à cet égard, Elsaesser a raison de dire que cette histoire croisée des médias et du cinéma - lequel est généralement arrimé aux seules histoires du regard et des images - est également concernée par les pratiques sonores issues de recherches effectuées dans les domaines du téléphone, de la radio et du disque ${ }^{5}$.

Des "premiers temps" à aujourd'hui, des vues Lumière à la consommation domiciliaire en réseaux, l'auteur parcourt ainsi transversalement l'histoire de l'histoire du cinéma - une histoire marquée par les chevauchements incessants et l'émergence souvent conjointe de "nouveaux médias» et de nouveaux modes de pensée. Chemin faisant, l'article rend compte à la fois de l'état du cinéma et de son histoire aujourd'hui. Surtout élaboré autour de l'inscription du cinéma dans une sphère médiatique 
très large, cet article est sans doute d'abord une méditation sur l'entre du cinéma - soit ce lieu d'intersections multiples d'une pratique intermédiale particulière. Là encore, un champ de recherche est grand ouvert, plein de promesses.

Par un télescopage un peu audacieux, la conclusion aurait ici très bien pu revenir à Dick Tomasovic qui, à propos du film d'animation, écrit à la fin de son article que les "nouvelles tendances médiatiques, toutes en relation avec le cinéma d'animation, [...] poussent les études filmiques et l'histoire du cinéma à se réinventer elles-mêmes". Car l'intention du responsable de ce numéro de CiNéMAS était aussi de conjuguer une réflexion sur l'écriture d'histoires croisées et sur l'étude d'objets particuliers. De fait, le film d'animation se prête bien au projet, au moins parce que cet objet singulier a historiquement ceci de fascinant qu'il révèle toujours quelque chose de l'état du cinéma. Fort sensible aux évolutions technologiques, l'animation paraît souvent avoir une longueur d'avance sur le cinéma. Du moins, ose-t-elle plus vite s'engager dans la foulée des «nouvelles images» et des "nouvelles technologies", et le cinéma de la suivre, parfois de l'imiter, pour reprendre à son compte des procédés «bâtards" venus d'un ailleurs jamais très distant. Cinéma vs animation? Tomasovic reprend cette relation duelle, parfois conflictuelle, pour suggérer une approche davantage distanciée et pour mieux "problématiser " ces "mélanges d'images" qui caractérisent autant l'histoire du cinéma que celle du film d'animation. Et cette histoire-là est une histoire où se croisent des images dont la résolution - tel un bouquet final étincelant, comme dans Fast Film, l'un des nombreux films ici évoqués - est de ré-inventer l'histoire du cinéma, ni plus, ni moins. "Pour y parvenir», ne manque pas d'écrire Tomasovic, "peut-être faudra-t-il diversifier davantage encore nos regards et conjuguer de plus en plus d'approches, particulièrement celles qui font des collisions leurs objets d'études spécifiques». Et cette histoire-là est, elle aussi, invariablement une histoire des images soucieuse d'épistémologie.

Valérie Pozner propose quant à elle une étude minutieuse consacrée aux "bonimenteurs rouges " des films soviétiques et à la question de l'oralité dans le cinéma muet. La perspective choisie par Pozner — dont on a récemment apprécié les 
recherches sur les «ciné-déclamateurs" russes — est tout autre ${ }^{6}$. Découvrir un pan entier de l'histoire du cinéma, absolument méconnu à ce jour, et "problématiser" son écriture, tel est l'objectif double de cette étude passionnante. Fruit de recherches d'une méticulosité rare, son travail s'inscrit à la suite des plus récentes analyses des pratiques sonores du cinéma muet - avec comme point de mire une étude de la réception et une lecture sociale de l'histoire du cinéma. Cet article partage néanmoins un dessein sans doute commun à l'ensemble des contributions ici réunies, soit penser l'histoire du cinéma autrement. Il n'est pas question d'autre chose quand la chercheuse écrit: "Dans le cas des "soirées thématiques", on a affaire à un spectacle véritablement intermédial, dans lequel un thème ou une situation narrative se voit décliné sous plusieurs formes, savantes et populaires, visuelles et verbales, écrites et parlées, autour de l'axe que constitue la projection cinématographique. "Enfin, Pozner, qui souligne au passage la valeur indicielle de la chronologie et ne manque pas d'évacuer ce qu'elle désigne par une "perspective auteuriste", n'hésite pas à affirmer les ambitions plus théoriques de son analyse ciselée, considérant que «le cas exposé doit peutêtre avant tout ouvrir sur une réflexion méthodologique».

Marquant les esprits de quelques grands philosophes et penseurs tout au long $\mathrm{du} \mathrm{XX}$ siècle, le septième art ne pouvait pas laisser plus longtemps indifférents les historiens qui, surtout sensibles aux seules traces du passé gardées par les films, considèrent dorénavant autrement l'importance de l'empreinte des images sur nos manières de faire, de voir et de penser. La réflexion de Carl Havelange, chercheur et enseignant en histoire culturelle, était à l'origine, comme l'indique l'auteur, une «réflexion introductive». Par la suite, il m’a semblé plus intéressant de faire une entorse au protocole initialement prévu, afin de placer sa note en continuité avec l'article conclusif de Sylvie Lindeperg. Tous les deux ont en effet dans leur ligne de mire une possible histoire du regard - qui est sans doute l'un des aboutissements sérieusement autorisés dès l'origine de ce numéro de CiNéMAS. Dans des styles proches et différents tout à la fois, s'intéressant à des objets en apparence assez éloignés, ces deux articles ont au moins une ambition commune, qui me 
paraît être l'une des voies les plus prometteuses aujourd'hui : approcher une histoire culturelle des images. C'est pourquoi je considère le détachement de Havelange face à l'image incertaine du cinéma comme sans doute feint en partie, eu égard à son intérêt prononcé pour les images et pour la photographie en particulier. Du moins me semble-t-il évident, à le lire, qu'une histoire culturelle des images passe invariablement par les histoires croisées, entre autres, de la photographie et du cinéma, d'autant plus qu'il s'agit aussi par là d'opérer une jonction forte entre la théorie et l'histoire, deux termes souvent disjoints — comme deux espaces arbitrairement inconciliables, au même titre que l'esthétique et les études historiques. Havelange l'écrit en effet avec conviction, et avec une justesse qui vaut d'être soulignée:

Question d'histoire, donc. Mais d'une histoire, évidemment, qui ne s'en tienne pas à la description des techniques, des genres et des styles, d'une histoire qui sache poser la question des fondements et finement évaluer les processus d'institutionnalisation et d' «invisibilisation" progressive qui sont à l'œuvre dans la production, la diffusion et la réception des images: une histoire culturelle des images. La théorie, dès lors — en ce beau sens étymologique du mot théorie qui postule l'exercice d'un regard —, n'adviendra plus comme en surplomb de l'histoire, mais au contraire comme une dimension essentielle de l'historicité qui, irréductiblement, qualifie les phénomènes culturels (p. 184-185).

Havelange et Lindeperg, travaillant dans des champs différents, ont fini par se rencontrer autour d'un projet commun, envisagé peut-être autrement mais accordant une place particulière aux images face à l'écriture historienne et convenant de la place cardinale de celles-ci face à la mémoire - entre perte et mélancolie, entre oubli et histoire. Or, la relation singulière de la mémoire à l'histoire est véritablement le centre névralgique de l'article final.

Si le délicat honneur de clore ce dossier de CiNéMAS revient à Lindeperg, c'est d'abord parce que son texte participe directement à un débat très soutenu qui porte conjointement sur les régimes d'historicité et sur la fonction des images. En la lisant, il est en effet difficile de ne pas songer aux plus récents ouvrages, 
entre autres de Georges Didi-Huberman, de François Hartog, de Paul Ricœur, ou, plus lointainement, de Louis Marin. Et de quelques autres encore: Jacques Revel, Pierre Laborie, tous deux cités; Paul Veyne et, surtout, Michel Foucault, en ombres projetées. Puis surgissent parfois quelques noms - ceux de Walter Benjamin et Gilles Deleuze — dont l'association est d'une richesse heuristique généralement mésestimée. Car si une première chose vient à l'esprit face à cet article — dont on se doit d'abord de souligner la valeur programmatique —, c'est bien d'avoir tissé un réseau de correspondances entre des pensées multiples. À propos de cette "histoire en rhizomes " (en référence à Gilles Deleuze), la tentation est grande de la décrire comme une pensée de l'histoire d'une envergure fort ample ${ }^{7}$. Dans le cadre d'une histoire croisée des images, l'article procède en vue de l'écriture "d'une autre histoire qui, s'intéressant aux interprétations successives de documents, proposerait une archéologie du travail de sédimentation qui les a fait migrer jusqu'à nous ".

Mémoire, histoire, oubli: l'article de Lindeperg ne manque pas au passage de s'inscrire en faux contre certaines dérives méthodologiques et éthiques engagées autour des pouvoirs de l'image et "des enjeux de re-figuration et de réécriture du passé $^{8}$ ». Car la réflexion excède le cadre parfois convenu de la mise en relation de l'histoire et du cinéma (ou de la photographie), pour davantage interroger les flux du passé qui marquent l'histoire et son écriture au présent:

En attachant une attention conjointe à l'image et à ses usages, il s'agit d'éclairer le mouvement dialectique qui va du passé vers le futur afin de placer en regard le contexte d'enregistrement du document et les conditions de son exhumation comme de ses réinterprétations successives (p. 200).

Enfin, Lindeperg accoste les rives d'une épistémologie de l'image, par l'intermédiaire d'incises au cœur des thèses sur l'histoire de Walter Benjamin — dont nous reviennent à l'esprit quelques-unes des propositions les plus fulgurantes pour aborder l'histoire comme construction ou mettre en réseaux des images d'hier, aujourd'hui. Dans ce "film palimpseste» inventé par Lindeperg, que l'on imagine fait de collages d'images et de 
montages d'extraits de films, le cinéma paraît effectivement en action, et son histoire, en construction. C'est notamment en cela que cette "mise en intrigue», comme la qualifie elle-même Lindeperg, constitue véritablement le programme d'une mémoire historienne inédite et une méthode d'écriture en vue d'une "histoire du regard» et des imaginaires collectifs. À l'évidence, une histoire à suivre de près.

La description globale qui ramène tout à son hypothétique point initial - en l'occurrence, le cinéma — paraît effectivement une vision désuète et du cinéma et de son histoire. Cette façon de voir paraît mener à une impasse. À force de négliger les mouvements constants situés aux arêtes extérieures du cinéma - sans cesse confronté aux "nouvelles images» et aux «nouvelles technologies», pris depuis ses origines entre les feux croisés de pratiques culturelles et médiatiques mixtes — cette histoire paraît indûment autosuffisante et sans réelle perspective. À l'inverse, une histoire croisée des images est assurément plurielle, à envisager comme une structure en mouvement, selon une diagonale mobile. De fait, les interstices où se croisent des images chaque fois singulières constituent à la fois un lieu de rencontre d'objets hétérogènes et un point d'impact de problématiques relatives à l'historiographie. C'est dire l'importance du chantier. En ce sens, l'ambition première à la base de ce numéro était bien de poser des balises et de s'immiscer entre les plis de l'histoire du cinéma et de son écriture. La proposition permettant de dire que l'histoire du cinéma et des images paraît aujourd'hui à la croisée de réflexions concernant autant le cinéma ou les images que l'écriture de leur histoire demeure valable. Et l'histoire et l'image ont certainement en commun de constituer deux modes particuliers de représentation. Or, comme l'a écrit Ricour (2000, p. 296), "présence, absence, antériorité, représentation forment la toute première chaîne conceptuelle du discours de la mémoire ${ }^{9} "$. Et ce discours, qui concerne directement l'histoire et la théorie du cinéma et des images, mérite sans nul doute d'être réévalué à l'avenir. La tâche est immense.

Aussi, ai-je envie de dire au terme de cette présentation qu'une histoire du cinéma et des images demeure en construction. 


\section{NOTES}

1. J'emprunte cette formule à Georges Didi-Huberman, qui a absolument raison d'ajouter que cette posture n'est pas sans risque car, dit-il plus précisément à propos de la démarche d'Aby Warburg, "excéder le cadre épistémologique de la discipline traditionnelle, c'était accéder à un monde ouvert de relations multiples, inouïes, dangereuses même à expérimenter» (Didi-Huberman 1998, p. 12).

2. Je pense à ses travaux consacrés à "Jarry et le cinéma", mais aussi aux recherches qu'elle effectue à l'Université de Lausanne aux côtés de François Albera. Je remercie au passage François Albera, mais aussi André Gaudreault, pour leur soutien tout au long du cheminement de ces Histoires croisées.

3. Récemment, Michel Frizot (2004) a articulé sa réflexion autour de ces "possibles du cinéma", non sans revenir sur les usages souvent abusifs du terme même d'archéologie et du sens très précis donné à ce mot par Michel Foucault. Frizot trouvera dans l'article de Tortajada de quoi largement se réconcilier avec une perspective archéologique dans le champ des études cinématographiques.

4. Larticle de Berton trouvera des alliés de taille dans cette manière (oblique et à sens multiples) d'envisager les liens unissant la psychanalyse à l'histoire ou à l'esthétique. Voir, entre autres: de Certeau 1975 et 1987; Deleuze 1972 et 1980; Rancière 2001.

5. Je souscris pleinement à cette hypothèse - sur laquelle il m'a été donné de m'attarder à plusieurs reprises. Voir à ce sujet mes deux ouvrages: Arnoldy 2004 et 2005.

6. Dans le cadre du colloque organisé par Valérie Pozner et Giusy Pisano au Louvre, Le muet a la parole (Paris, Auditorium du Louvre, 12-13 juin 2004, actes à paraître courant 2005).

7. Lors d'un colloque à l'INHA en novembre 2002, j'ai moi-même émis l'hypothèse d'une telle "histoire en rhizomes", avec pour point de départ la dimension organique de l'archéologie discursive développée par Michel Foucault — notamment dans son exposé "La volonté de savoir" - , non sans songer à Deleuze et aux liens forts qui ont uni les deux philosophes. Voir Foucault 2001 et Arnoldy $2004 a$.

8. La confrontation des choix posés ici et de ceux de Didi-Huberman dans Images malgré tout (2003) mériterait à elle seule de plus longs développements. Le débat est ouvert.

9. Sur cette question d'une "histoire-représentation", trop vaste pour être développée ici, je renvoie à deux ouvrages en particulier : Marin 1993 et Ricœur 2000.

\section{RÉFÉRENCES BIBLIOGRAPHIQUES}

Arnoldy 2004 : Édouard Arnoldy, Pour une histoire culturelle du cinéma. Au-devant de "scènes filmées", de "films chantants et parlants" et de comédies musicales, Liège, Céfal, 2004.

Arnoldy 2004a : Édouard Arnoldy «De la rumeur infinie des archives. Questions posées aux fins d'une histoire de la production culturelle», dans Irène Bessière et Jean A. Gili (dir.), Histoire du cinéma. Problématique des sources, Paris, Institut National d'Histoire de l'Art/AFRHC, 2004, p. 27-39.

Arnoldy 2005 : Édouard Arnoldy, À perte de vues. Images et "nouvelles technologies", d'hier et d'aujourd'hui, Bruxelles, Labor, 2005.

de Certeau 1975: Michel de Certeau, «Écritures freudiennes» [1970], L’Écriture de l'histoire, Paris, Gallimard, 1975, p. 291-358. 
de Certeau 1987: Michel de Certeau, Histoire et Psychanalyse, entre science et fiction, Paris, Gallimard, 1987.

Deleuze 1972: Gilles Deleuze, L'Anti-CEdipe, Paris, Minuit, 1972.

Deleuze 1980 : Gilles Deleuze, Mille Plateaux, Paris, Minuit, 1980.

Didi-Huberman 1998: Georges Didi-Huberman, «Savoir-Mouvement (L'Homme qui parlait aux papillons)", dans Philippe-Alain Michaud, Aby Warburg et l'image en mouvement, Paris, Macula, 1998, p. 7-20.

Didi-Huberman 2003: Georges Didi-Huberman, Images malgré tout, Paris, Minuit, 2003.

Foucault 2001: Michel Foucault, "La volonté de savoir», Annuaire du Collège de France, $71^{\circ}$ année, Histoire des systèmes de pensée, année 1970-1971 [1971], dans Dits et écrits, 1954-1975, volume I, Paris, Gallimard, 2001, p. 1108-1112.

Frizot 2004 : Michel Frizot, "Qu'est-ce qu’une invention? (le cinéma). La technique et ses possibles", Trafic, n' 50, 2004, p. 316-326.

Marin 1993: Louis Marin, Des pouvoirs de l'image, Paris, Seuil, 1993.

Rancière 2001 : Jacques Rancière, L'Inconscient esthétique, Paris, Galilée, 2001.

Ricœur 2000 : Paul Ricœur, La Mémoire, l'Histoire, l'Oubli, Paris, Seuil, 2000. 